\title{
Publisher's Note: Enhancing Coherent Light-Matter Interactions through Microcavity-Engineered Plasmonic Resonances [Phys. Rev. Lett. 119, 233901 (2017)]
}

Pai Peng (彭看), Yong-Chun Liu, Da Xu, Qi-Tao Cao, Guowei Lu, Qihuang Gong, and Yun-Feng Xiao

(Q) (Received 27 December 2017; published 4 January 2018)

DOI: 10.1103/PhysRevLett.120.019901

This Letter was published online on 4 December 2017 with an incorrect address for an affiliation. The third affiliation should read as “3 Collaborative Innovation Center of Extreme Optics, Shanxi University, Taiyuan, 030006 Shanxi, People's Republic of China." The affiliation has been corrected as of 28 December 2017. The affiliation is incorrect in the printed version of the journal. 